\title{
Biodiesel trace element analysis by energy dispersive $X$-ray fluorescence spectrometry using magnetic solid phase microextraction
}

Lucilia A. Meira ${ }^{1}$, Jorge S. Almeida ${ }^{1,2}$, Maiara S. Oliveira ${ }^{1}$, Fabio de S. Dias ${ }^{3}$, Antonio F. S. Queiroz ${ }^{4}$, Sarah A. R. Soares ${ }^{4}$, Leonardo S. G. Teixeira ${ }^{1,2 *}$

${ }^{1}$ Universidade Federal da Bahia, Instituto de Química, Departamento de Química Analítica, Campus Universitário de Ondina, 40170-115, Salvador, Bahia, Brazil

${ }^{2}$ INCT de Energia e Ambiente - Universidade Federal da Bahia, Instituto de Química, Campus Universitário de Ondina, 40170-115, Salvador, Bahia, Brazil

${ }^{3}$ Instituto de Ciências Tecnologia e Inovação, Universidade Federal da Bahia, Campus Universitário de Camaçari, Camaçari, Bahia, 42809-000, Brazil

${ }^{4}$ Instituto de Geociências, Universidade Federal da Bahia, Campus Universitário de Ondina, Salvador, Bahia 40170-115, Brazil 


\section{Supplementary Information}

Table S1 shows the analysis of variance (ANOVA) for the linear and quadratic models used to evaluate the behavior of the experimental data obtained through the constraint mixing design. The total error associated with the linear model was larger than that obtained by the quadratic model. The large residues associated with the linear model can result in a lower predictive capacity for the model. Thus, the quadratic model was choice, resulting in smaller errors.

Table S1- Analysis of variance (ANOVA) for linear and quadratic models (at 95\% confidence level).

\begin{tabular}{llllll}
\hline Variation source & SS & Df & MS & Fcrit & P-value \\
\hline Linear model & & & & & \\
\hline Model & 3.509459 & 2 & 1.754730 & 2.265496 & 0.174305 \\
Total error & 5.421819 & 7 & 0.774546 & & \\
Total adjusted & 8.931278 & 9 & 0.992364 & & \\
\hline Quadratic model & & & & & \\
Model & 6.539339 & 5 & 1.307868 & 2.187125 & 0.234143 \\
Total error & 2.391939 & 4 & 0.597985 & & \\
Total adjusted & 8.931278 & 9 & 0.992364 & & \\
\hline
\end{tabular}

SS: sum of square; df: degrees of freedom; MS: mean square 